\section{Evaluation of biofilm forming capacity of canine otic isolates of Malassezia pachydermatis (MP)}

\author{
Stephen Steen ${ }^{1}$, Susan Paterson ${ }^{2}$ \\ 1 CAPL, Keele, United Kingdom \\ 2 Virtual Vet Derms, Kendal, United Kingdom
}

\section{OBJECTIVES}

This study was designed to assess the biofilm forming capacity of 10 isolates of Malassezia pachydermatis taken from clinical cases of canine otitis to assess if this may be the reason for treatment failure with topical antimycotic drugs.

\section{METHODS}

M. P. isolates from clinical cases submitted to the laboratory, were identified by their microscopic and macroscopic morphology as well as their ability to grow on
Sabouraud's dextrose agar. Biofilms were produced using a variety of methods (Pye 2013, Figueredo 2012 ). Each isolate was categorised as a non, low, medium or high biofilm producer based on their optical density measured at $570 \mathrm{~nm}$. In view of the small number of isolates that were assessed, statistical analysis was not considered appropriate and was not undertaken.

Pye CC et al. Evaluation of biofilm production by Pseudomonas aeruginosa from canine ears and impact of biofilm on antimicrobial susceptibility in vitro. Vet Dermatol 2013, 24:446-449

Figueredo LA et al Antifungal susceptibility of Malassezia pachydermatis biofilm Med Mycol, 2013, 51, 863-867

\section{RESULTS}

All 10 isolates assessed for biofilm production were found to be high level producing strains. In view of the fact that the antifungal susceptibility of Malassezia is known to be decreased when biofilm is present these results may explain the reason for treatment failure.

\section{STATEMENT (CONCLUSIONS)}

Where infection with Malassezia pachydermatis fails to respond to antimycotic therapy in cases of otitis externa it is possible that biofilm may be present. 